\title{
Wirtschaftspolitik als Institutionendesign: eine Einführung
}

\author{
Mathias Erlei
}

Online publiziert: 13. November 2017

(C) List-Gesellschaft e.V. 2017

Das Institutionendesign ist seit jeher ein zentraler Bestandteil der Wirtschaftspolitik in allen Wirtschaftssystemen. Institutionen schränken den Handlungsspielraum von bestimmten privaten und öffentlichen Akteuren ein, erzeugen damit neue Handlungsfreiräume bei anderen Entscheidungsträgern und setzen zugleich Anreize für Verhaltensweisen, die als gesellschaftlich wünschenswert angesehen werden. Aus diesen Gründen hat sich der Ausschuss für Wirtschaftspolitik (im Verein für Socialpolitik) auf seiner Jahrestagung mit dem Oberthema „Wirtschaftspolitik als Institutionendesign“ beschäftigt. Das vorliegende Sonderheft enthält die auf dieser Tagung gehaltenen wissenschaftlichen Vorträge. ${ }^{1}$

Im ersten Beitrag beschäftigt sich Jan Schnellenbach aus verhaltensökonomischer Sicht mit Problemen politischer Entscheidungsverfahren. Der Ansatzpunkt des Aufsatzes besteht darin, dass Politiker nicht nur den vielfach untersuchten Interessenund Kontrollproblemen unterliegen, sondern dass ihre Entscheidungen natürlich auch von den normalen menschlichen Entscheidungsverzerrungen und Rationalitätsmängeln, wie sie in der Verhaltensökonomik untersucht werden, behaftet sind. Hierauf aufbauend argumentiert er, dass die Delegation politischer Entscheidungen an politische Entscheidungsträger keineswegs als Substitut für die fehlende Rationalität der Bürger dienen kann. Vielmehr bedarf es eines offenen und wettbewerblichen politischen Entscheidungsverfahrens, das die systematischen Entscheidungsverzerrungen der Individuen noch am besten einschränkt. Außerdem liefert der Verfasser weitere Gründe dafür, dass bewährte Institutionen, wie z. B. Checks-and-balances

\footnotetext{
1 Ein Beitrag, den die in der Praxis tätigen Autoren Schackmann-Fallis und Weiß in der Arbeitsgruppe Wettbewerb vorgestellt haben, wurde ohne Begutachtungsverfahren in das Sonderheft aufgenommen.
}

M. Erlei (殴)

Technische Universität Clausthal, Clausthal-Zellerfeld, Deutschland

E-Mail: m.erlei@tu-clausthal.de 
oder die Regelbindung der Politik, positive Beiträge zur Vermeidung von Entscheidungsverzerrungen liefern. Schließlich plädiert er für die Einrichtung unabhängiger Organisationen, die Wahrnehmungsverzerrungen in öffentlichen Diskursen durch Aufklärungsmaßnahmen entgegenwirken.

Oliver Budzinski untersucht die Auswirkungen der Digitalisierung auf das moderne Wettbewerbsrecht. Insbesondere widmet er sich der Frage, inwieweit die neunte Novelle des Gesetzes gegen Wettbewerbsbeschränkungen diesen Anforderungen genügt. Im Vordergrund des Beitrags stehen drei Aspekte der Digitalisierung der Wirtschaft: (1) Die Substitution von Geldpreisen durch das Zahlen mit Daten (Null-PreisÖkonomie); (2) Wettbewerb zwischen Plattformbetreibern (zweiseitige Märkte) und (3) die Kopplung des Wettbewerbsrecht mit dem Verbraucherschutz. Für alle drei Gebiete zeigt der Verfasser, dass die Novelle gewisse Verbesserungen erreicht hat, andere Probleme aber noch ungelöst sind. Besonders kritisch wird die datenbasierte individualisierte Preisdiskriminierung gesehen, bei der ein per se-Verbot vorstellbar sei. Abschließend werden weitere Aspekte der Digitalisierung - wie etwa die algorithmenbasierte Kollusion - angerissen, die noch nicht im Fokus der GWB-Novelle liegen.

Martina Eckardt und Stefan Okruch analysieren den Europäischen Verbund für territoriale Zusammenarbeit (EVTZ), der die Einrichtung von grenzüberschreitenden Organisationen zur Verbesserung der Governance territorialer Zusammenarbeit (insb. von Gebietskörperschaften und Einrichtungen des öffentlichen Rechts) fördern soll. Fruchtbare Ansatzpunkte für solche Organisationen sind unter anderem die grenznahe Bereitstellung von Infrastruktur und die Vertiefung der wirtschaftlichen Integration. Erste Erfahrungen mit dem relativ neuen Instrument sind positiv, vor allem im Hinblick auf die Stärkung grenznaher Problemregionen.

Thomas Grebel untersucht den Einfluss staatlicher F\&E-Förderung auf die privatwirtschaftliche Forschungs- und Entwicklungsaktivität. Anhand eines anreizorientierten Duopolmodells wird gezeigt, dass sich die staatliche F\&E-Förderung positiv auf die private F\&E-Tätigkeit auswirkt, falls sie symmetrisch angelegt ist, d. $h$. falls sie allen Unternehmen gleichermaßen zugutekommt. Eine asymmetrische F\&EFörderung induziert Verzerrungen, die unter bestimmten Bedingungen dazu führen können, dass die private F\&E-Aktivität verringert wird. Dementsprechend sollte die staatliche Förderpolitik die zu erwartenden Verdrängungseffekte hinreichend berücksichtigen.

Peter Spahn diskutiert die institutionelle Ausgestaltung der Zentralbank in einer Währungsunion im Hinblick auf die Absicherung der Staatsschulden. Er zeigt auf, dass im Fall eines Verzichts der Zentralbank auf die Lender-of-Last-Resort-Funktion (LLR-Funktion) die Existenz multipler Gleichgewichte zu einer Staatsschuldenkrise selbst für relativ gering verschuldete Länder führen kann. Greift die Zentralbank hingegen helfend ein, so erzeugt sie ein Moral-Hazard-Problem, das die Länder zu höherer Verschuldung treibt. Da die Zentralbank einer Währungsunion kein Mandat zu einer fiskalpolitischen Umverteilung hat, die mit der Hilfe des Schuldenmanagements von ausgewählten Mitgliedstaaten einhergeht, kann die LLR-Funktion nur für das Währungsgebiet als Einheit erbracht werden. Die Teilstaaten müssten - dem Vorbild der USA entsprechend - eigenverantwortlich handeln. Die Rückkehr zu einer wahren No-Bailout-Strategie erfordert jedoch einen vorherigen Schuldenschnitt. 
Horst Gischer, Christian Ilchmann und Toni Richter thematisieren das Spannungsverhältnis zwischen zwei Grundpfeilern der Europäischen Union - der (solidarischen) „Vereinigung in Vielfalt“ und dem Subsidiaritätsprinzip - anhand der Bankenunion, insbesondere der geplanten einheitlichen Einlagensicherung. Im Gegensatz zur Einlagensicherungsrichtlinie aus dem Jahr 2014 enthalten die neueren Überlegungen zu einer einheitlichen Einlagensicherung nur einen geringen Bezug zum Subsidiaritätsprinzip, sodass die Risiken von Einlagenverlusten damit kaum wirkungsvoll gesenkt werden können. Um eine Vergemeinschaftung von Risiken zu vermeiden, schlagen die Autoren vor, den grenzüberschreitenden Ausgleich von Fondsrisiken über ein freiwilliges Rückversicherungssystem zu realisieren.

Karl-Peter Schackmann-Fallis und Mirko Weiß analysieren die Rolle der Sparkassen auf dem deutschen Bankenmarkt. Dabei beschreiben sie die besonderen Funktionen des Sparkassensystems im Hinblick auf die Finanzierung kleiner und mittelgroßer Unternehmen, ihren öffentlichen Auftrag, insbesondere zur Daseinsvorsorge, zur regionalen Ausrichtung und zur Finanzierung des Infrastrukturausbaus. Durch politische Entscheidungen wie die zunehmenden Regulierungsanforderungen, die europäische Bankenabgabe und die drohenden Belastungen durch eine europäische Einlagensicherung entsteht eine erhebliche Belastung vor allem kleinerer und mittlerer Institute. Aus diesem Grund wird eindringlich vor Umsetzung der Risikovergemeinschaftung durch die diskutierte europäische Einlagensicherung gewarnt. 